\title{
Teres Ligament Patch Reduces Relevant Morbidity After Distal Pancreatectomy (the DISCOVER Randomized Controlled Trial)
}

\author{
Matthias Hassenpflug, MD, ${ }^{*}$ Ulf Hinz, MSc, ${ }^{*}$ Oliver Strobel, MD, ${ }^{*}$ Johanna Volpert, MD, ${ }^{*}$ \\ Philip Knebel, MD, ${ }^{*} \dagger$ Markus K. Diener, MD, ${ }^{*} \dagger$ Colette Doerr-Harim, MD, $\dagger$ Jens Werner, MD, $\ddagger$ \\ Thilo Hackert, MD, ${ }^{*}$ and Markus W. Büchler, MD*
}

\begin{abstract}
Objective: The aim of this study was to analyze the impact of teres ligament covering on pancreatic fistula rate after distal pancreatectomy (DP).

Background: Postoperative pancreatic fistula (POPF) represents the most significant complication after DP. Retrospective studies suggested a benefit of covering the resection margin by a teres ligament patch.

Methods: This prospective randomized controlled study (DISCOVER trial) included 152 patients undergoing DP, between October 2010 and July 2014. Patients were randomized to undergo closure of the pancreatic cut margin without (control, $\mathrm{n}=76$ ) or with teres ligament coverage (teres, $\mathrm{n}=76$ ). The primary endpoint was the rate of POPF, and the secondary endpoints included postoperative morbidity and mortality, length of hospital stay, and readmission rate.

Results: Both groups were comparable regarding epidemiology (age, sex, body mass index), operative parameters (OP time, blood loss, method of pancreas transection, additional operative procedures), and histopathological findings. Overall inhospital mortality was $0.6 \%$ (1/152 patients). In the group of patients with teres ligament patch, the rate of reoperations $(1.3 \%$ vs $13.0 \%$; $P=0.009)$, and also the rate of readmission (13.1 vs $31.5 \% ; P=0.011)$ were significantly lower. Clinically relevant POPF rate (grade B/C) was $32.9 \%$ (control) versus $22.4 \%$ (teres, $P=0.20$ ). Multivariable analysis showed teres ligament coverage to be a protective factor for clinically relevant POPF $(P=$ 0.0146).

Conclusions: Coverage of the pancreatic remnant after DP is associated with less reinterventions, reoperations, and need for readmission. Although the overall fistula rate is not reduced by the coverage procedure, it should be considered as a valid measure for complication prevention due to its clinical benefit.
\end{abstract}

Keywords: coverage, covering technique, distal pancreatectomy, morbidity, pancreatic fistula, teres ligament patch

(Ann Surg 2016;264:723-730)

From the *Department of General, Visceral and Transplantation Surgery; $†$ Study Center of the German Surgical Society (SDGC), University of Heidelberg, Heidelberg, Germany; and $\ddagger$ Department of General, Visceral, and Transplant Surgery, Ludwig-Maximilians-University, Munich, Germany.

Reprints: Markus W. Büchler, MD, Department of Surgery, University of Heidelberg, Im Neuenheimer Feld 110, 69120 Heidelberg, Germany.

E-mail: Markus_Buechler@med.uni-heidelberg.de.

Supplemental digital content is available for this article. Direct URL citations appear in the printed text and are provided in the HTML and PDF versions of this article on the journal's Web site (www.annalsofsurgery.com).

Authors' contribution: Matthias Hassenpflug was writing the manuscript and was aided by Markus Büchler, Thilo Hackert, Markus Diener, and Oliver Strobel. Philip Knaebel was helping in conducting the trial together with Jens Werner. Ulf Hinz cared for the statistics. Johanna Volpert and Colette Doerr-Harim were screening patients and were performing the follow-up.

Conflicts of interest and source of funding: All authors declare not to have any conflicts of interest. There was no funding for the DISCOVER trial.

Copyright (C) 2016 Wolters Kluwer Health, Inc. All rights reserved.

ISSN: 0003-4932/16/26405-0723

DOI: $10.1097 /$ SLA.0000000000001913
D ancreatic resections can be carried out with low mortality in highvolume institutions today, ${ }^{1}$ but postoperative pancreatic fistula (POPF) remains the most important reason for postoperative morbidity. Although distal pancreatectomy (DP) is a technically rather simple procedure in most cases, safe closure of the pancreatic remnant persists to be a main issue of this operation. The frequency of POPF was described to be $35 \%$ in large series. ${ }^{2,3}$ During the past decade, several surgical techniques for secure closure of the pancreatic remnant failed to decrease this high incidence. ${ }^{4-7}$ One randomized trial and 4 retrospective series, ${ }^{5,8-11}$ including 1 prospective study of the authors' institution ${ }^{12}$ that were using coverage procedures, have shown encouraging results with regard to POPF prevention.

The aim of the DISCOVER trial was to evaluate the technique of remnant-coverage by use of a teres ligament patch for preventing POPF and relevant complications in a monocentric randomized controlled trial.

\section{METHODS}

The protocol of the DISCOVER trial was registered (German Clinical Trials Register: DRKS00000546) and published to ensure the transparency of the design and analysis procedures, after approval by the Ethics Committee of the University of Heidelberg. ${ }^{17}$ The trial was conducted at the Clinical Trial Center (KSC) of the Department of General, Visceral, and Transplantation Surgery at the University Hospital Heidelberg. The statistical design, data management, and analysis were performed independently at the Institute of Medical Biometry and Informatics (IMBI) of the University of Heidelberg.

\section{Participants}

Adult patients planned for elective, open DP for benign or malignant tumors, chronic pancreatitis, or pseudocysts of the pancreatic body or tail were eligible after giving informed consent. The exclusion criteria were participation in another interventional trial, current immunosuppressive therapy, or lack of compliance. ${ }^{17}$

\section{Randomization}

After they had given informed consent, the patients were randomized intraoperatively after confirming the indication for DP by the attending surgeon. Unstratified block randomization was performed with random block sizes in a 1:1 allocation ratio. A random list was generated using the PROC PLAN feature of SAS software (Cary, NC) by the IMBI. The random allocation sequence was implemented by the use of sequentially numbered opaque envelopes. The randomization process and assignment of participating patients to the trial intervention were performed by staff members of the KSC.

\section{Intervention}

After laparotomy and exploration of the abdominal cavity, assessing resectability and randomization, the pancreatic body and tail were mobilized and the transection site was defined. Afterwards, 
transection was performed by scalpel (fish-mouth technique) or stapler based on the observation that both of these methods are equally effective with regard to POPF rate. ${ }^{6}$ In case of scalpel transection, the pancreatic duct was identified and closed by crossing stitches. The dorsal and ventral edges of the resection margin were adapted using single stitches. Use of fibrin glue, meshes, or other additional methods for closing or covering of the pancreatic remnant was not allowed. An additional splenectomy or cholecystectomy was based on the surgeon's decision. Extended DP (vascular/additional organ resection) did not conflict with the protocol.

In the control group, DP was performed as described above without further coverage of the pancreatic remnant.

In patients randomized for coverage of the remnant pancreas, the teres ligament, together with the surrounding falciform ligament, was completely mobilized from the abdominal wall, brought to the resection margin through an opening of the lesser omentum, and fixed by an anterior and posterior suture line to entirely cover the pancreatic cut margin.

At the end of the operation, 2 passive drainage tubes were placed at the pancreatic remnant. Drainages were removed if the amount of fluid did not exceed $200 \mathrm{~mL} / \mathrm{d}$, pancreatic enzyme concentrations had normalized, and no bile or blood was present in the fluid. All drains were kept in situ for at least 3 days postoperatively.

\section{Objectives and Outcomes}

The primary endpoint was the occurrence of POPF (any grade) as per the definition of the International Study Group on Pancreatic Fistula (ISGPF). ${ }^{13}$ Briefly, grade A fistulas were defined as clinically asymptomatic without any further therapeutic consequences or POPF-related complications. Patients with parenteral nutrition, antibiotics, endoscopic stenting, or need for readmission were classified as grade B. Patients requiring a POPF-associated interventional drainage postoperatively were classified as grade C. ${ }^{14}$ Patients with POPF-associated postoperative bleeding [postpancreatectomy hemorrhage $(\mathrm{PPH})]$ leading to angiographic or surgical reintervention, and also all other POPF-associated reoperations were also classified as grade $\mathrm{C}$. The interventional POPF management was standardized in both groups and depended on the individual course.

Secondary endpoints were operation time, selected postoperative complications [wound infection, delayed gastric emptying (DGE) ${ }^{15} \mathrm{PPH},{ }^{16}$ abdominal rupture, intra-abdominal collections, operative or interventional revisions], inhospital mortality, duration of hospital stay, and need for readmission. Detailed definitions of the secondary endpoints are given in the protocol. ${ }^{17}$ An additional analysis of treatment costs that also considered readmissions was performed. The information of treatment costs was provided by the financial controlling department of the authors' institution.

Assessment of endpoints, adverse events (AEs), and serious AEs (SAEs) was done during postoperative ward visits by members of the KSC of the surgical department. On postoperative day 40, all patients underwent a phone interview to obtain information about further treatment or need for readmission elsewhere. In a final follow-up 1 year postoperatively, the local hospital patient database was searched for readmissions or reoperations associated with the initial operation.

\section{Sample Size Calculation}

The study was designed as a monocenter, randomized controlled superiority trial with 2 parallel treatment arms comparing an additional reinforcement of the pancreatic remnant by coverage with a teres ligament patch versus stapler or suture closure alone for preventing POPF after elective DP. The expected POPF rate of $16 \%$ in the experimental group (teres ligament patch) was based on the results of 1 randomized trial and 4 retrospective case series ${ }^{5,8-11}$ in which coverage of the pancreatic remnant was performed and the internationally accepted definition of POPF according to ISGPF was used. The POPF rate in the control group was expected to be $36 \%$ based on the 30-day follow-up results of the large multicenter, randomized DISPACT trial in which no group received additional coverage. ${ }^{6}$ With alpha $=5 \%$ and beta $=20 \%$, a sample size of $\mathrm{n}=75$ per group was necessary to detect a difference between the 2 groups when the chi-square test (2-sided analysis) is used. In addition, a logistic regression model including covariates of prognostic importance [age, body mass index (BMI), and extent of resection] was applied in confirmatory analysis.

\section{Statistical Analysis}

Final analyses were done in accordance with the intentionto-treat principle. A binary logistic regression model was applied to compare POPF rates in the 2 groups, adjusting for age, BMI, and extent of resection. The secondary endpoints were analyzed descriptively by calculation of adequate measures of the empirical distribution, and also descriptive $P$ values (result of $t$ tests in case of continuous data, of chi-square tests in case of categorical data) and associated $95 \%$ confidence intervals (CIs). A post hoc analysis was done to detect possible risk factors affecting the development of a pancreatic fistula. A binary logistic regression model was used with primary endpoint as a dependent variable. Two-sided $P$ values were used throughout (SAS Version 9.4, SAS Institute Cary, NC). If DP was not possible or a total pancreatectomy was performed after randomization, these patients were excluded from the analysis because the primary endpoint was not assessable.

\section{RESULTS}

\section{Patient Enrollment}

Between October 2010 and July 2014, 161 patients were randomized. Nine patients had to be excluded because inclusion criteria were not met after randomization. Thus, the modified intention-to-treat population consisted of 152 patients (coverage procedure $n=76$, controls $n=76$; Fig. 1). Baseline characteristics were equally distributed between both groups (Table 1).

\section{Primary Endpoint}

Patients without additional coverage after DP developed a POPF of any grade in 39 cases $(51.3 \%)$, whereas patients randomized for additional coverage with teres ligament patch developed a POPF of any grade in 36 cases (47.4\%). No significant difference could be detected in the univariate analysis with the chi-square test $(P=0.6265)$. The binary logistic regression model adjusting for age, BMI, and extent of resection showed younger patients to be at a higher risk for POPF development $(P$ $=0.03)$, whereas the coverage procedure was not a significant factor [odds ratio (OR) $0.821,95 \%$ CI $0.38-1.773$ ].

Clinically relevant POPF (grade B/C) occurred in 17 patients with and 25 patients without coverage procedure (grade $B: n=6$ vs $n$ $=4$; grade $\mathrm{C}: \mathrm{n}=11$ vs $\mathrm{n}=21$ ), resulting in a $10 \%$ reduction of grade $\mathrm{B} / \mathrm{C}$ POPF by teres ligament coverage $(P=0.1468$; Fig. 2$)$.

\section{Secondary Endpoints}

Patients without coverage had significantly more postoperative reinterventions (interventional drainage procedure, angiographic bleeding control, endoscopy for stent placement, operative revision; $35.5 \%$ vs $19.7 \%$; $P=0.03$; Table 2 ). These differences were even more pronounced in the subgroup analysis of POPF patients (Table 3). Seven out of 9 reoperated controls with POPF had fistula-related reasons for revision, whereas only 1 patient from the study group had to be reoperated due to fistulaassociated complications. 


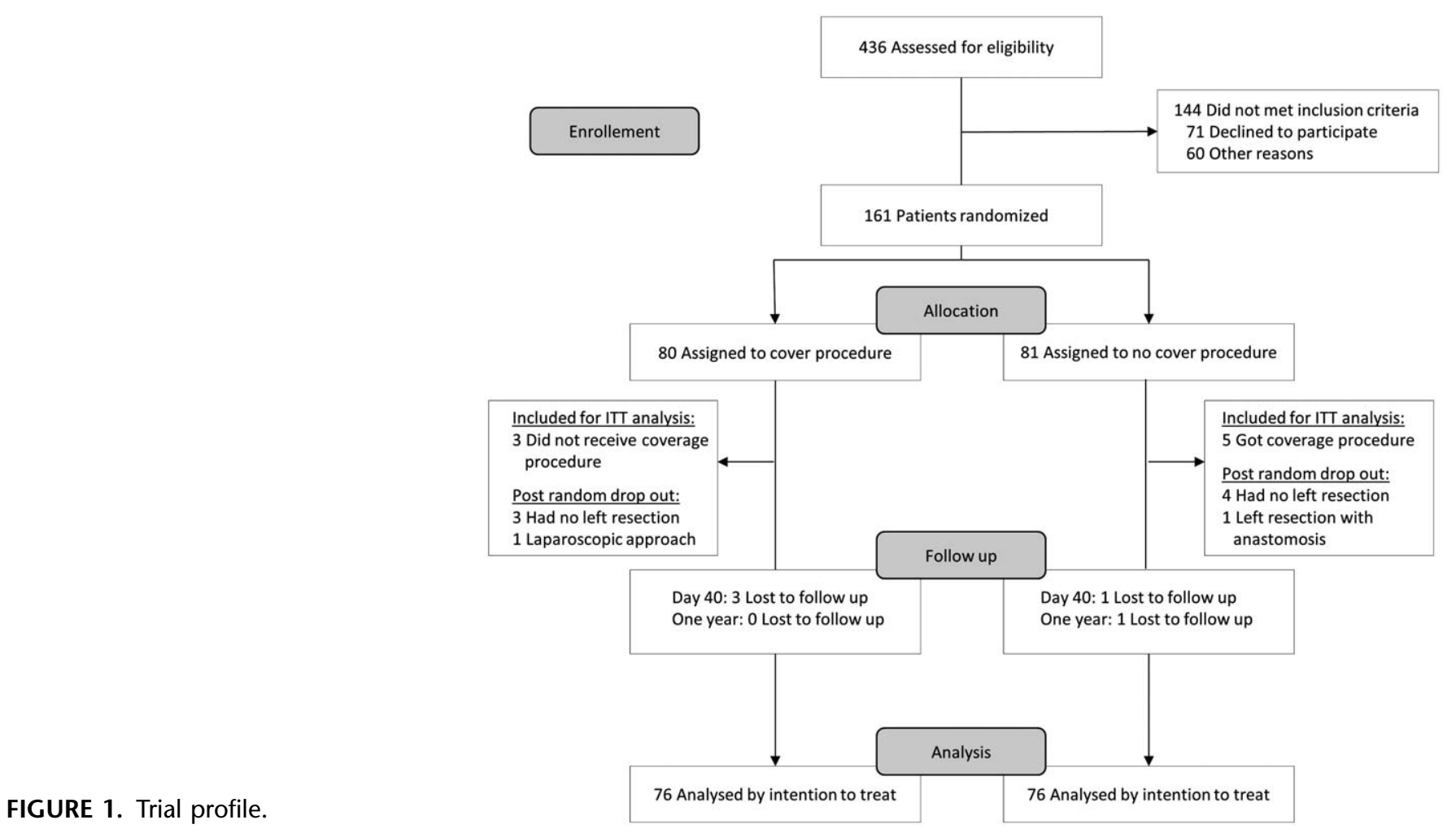

Readmission was necessary significantly more often in control patients (31.6\% vs $13.2 \%$; $P=0.006)$, including 20 readmissions later than postoperative day 40 (Table 2).

There were no differences in operation time, the rate of wound infections, DGE, PPH, and incidence of abdominal rupture. Overall mortality was $0.6 \%$, with 1 patient in the control group who died after apoplectic stroke on postoperative day 14 (Table 2).

Mean amylase levels and mean volume of drainage fluid showed no differences on postoperative days 1 to 4 between both groups. When comparing the maximum amylase value, covered patients showed significantly lower values $(7444$ vs $18083 \mathrm{U} / \mathrm{L} ; P$ $=0.011)$. Furthermore, in patients who required interventional drain placement, maximum amylase values were significantly lower in covered patients in the fluid obtained during drainage (15321 vs $55203 \mathrm{U} / \mathrm{L} ; P=0.03$ ).

Exploratory multivariable analysis including potential risk factors for POPF confirmed that age $(P=0.0055)$, length of hospital stay $(P=0.0149)$, and a resection level left of the portal vein correlated with an increased risk of POPF $(P=0.0448$; Supplementary material, Table 4, http://links.lww.com/SLA/B59).

Analysis of factors associated with POPF grade B and C, performed by a stepwise logistic regression analysis with a forward selection of correlating factors, revealed that the coverage procedure $(P$ $=0.0146)$ was a protective factor from developing a POPF of grade B or C (Supplementary material, Table 5, http://links.lww.com/SLA/B59).

Total inpatient treatment costs were calculated including expenses for readmissions. Analysis of POPF patients revealed significantly lower median treatment costs in patients with coverage compared with controls (Table 3).

\section{DISCUSSION}

Distal pancreatectomy is associated with low mortality, but POPF remains the most important complication with an incidence of approximately $35 \%,{ }^{2,3}$ with potential consequences for patients in terms of the need for reinterventions, length of hospital stay, and readmissions. In addition, these complications are a relevant burden in terms of healthcare costs. ${ }^{12,18}$

In the present randomized controlled trial, the effect of coverage of the pancreatic remnant with a teres ligament patch on relevant clinical complications-including POPF-was investigated. The teres ligament patch is an easily applicable surgical procedure during DP. The postoperative course revealed major differences between patients with and without coverage, with significantly more operative and nonoperative reinterventions, and also readmissions when no coverage was performed. Although the primary endpoint of the study (POPF incidence) did not show significant differences between the groups, the multivariable analysis revealed that the coverage procedure was a protective factor with regard to clinically relevant POPF development. This observation raises the question, if POPF incidence is an adequate primary endpoint in the design of surgical studies on DP, although it has been widely used in the past. ${ }^{5,6,8,11,12}$ The present study shows that other endpoints (ie, reintervention, readmission) are highly relevant for patient outcome as well, and that the more complicated postoperative course of fistula patients without coverage procedure does not result in a significantly different distribution of fistula grades, rated by the ISGPF definition. ${ }^{13}$ Especially with regard to grade $\mathrm{C}$ POPF, this becomes evident as patients with POPF-associated prolonged intensive care treatment and need for recurrent surgery cannot be distinguished from patients receiving interventional drainage without further complications as a very common type of management in grade C POPF. Both types of management fulfill the prerequisites for grade $\mathrm{C}$ according to the current definition which was used in the present study. A proposed adaptation of the fistula definition rating interventional managed cases as grade B could help to improve accuracy of the clinical grading in these patients. ${ }^{14}$ 
TABLE 1. Patient Characteristics (mITT)

\begin{tabular}{|c|c|c|c|c|}
\hline & Coverage $(n=76)$ & No coverage $(n=76)$ & Total $(N=152)$ & $\boldsymbol{P}$ \\
\hline Age (yrs, median, range) & $65(29-85)$ & $67.5(35-85)$ & $66(29-85)$ & 0.119 \\
\hline Sex & & & & 1 \\
\hline Male & $34(44.7 \%)$ & $34(44.7 \%)$ & $68(44.7 \%)$ & \\
\hline BMI, $\mathrm{kg} / \mathrm{m}^{2}$ & 24.7 & 25.8 & 25.1 & 0.418 \\
\hline \multicolumn{5}{|l|}{ Indication for operation } \\
\hline Malignant tumor & $29(38.2 \%)$ & $34(44.7 \%)$ & $63(41.4 \%)$ & 0.414 \\
\hline Others & 2 & 6 & 8 & \\
\hline Benign tumor & $35(46.1 \%)$ & $31(40.8 \%)$ & $66(43.4 \%)$ & 0.516 \\
\hline Cystadenoma & 6 & 11 & 17 & \\
\hline IPMN & 6 & 10 & 16 & \\
\hline Benign neuroendocrine & 16 & 6 & 22 & \\
\hline Others & 7 & 4 & 11 & \\
\hline VC (compared with age-matched group) & $93 \%$ & $88.7 \%$ & $90.1 \%$ & 0.994 \\
\hline FEV1 (compared with age-matched group) & $95.7 \%$ & $95.8 \%$ & $95.8 \%$ & 0.635 \\
\hline History of smoking & $38(50 \%)$ & $36(47.4 \%)$ & $74(48.7 \%)$ & 0.746 \\
\hline Ongoing smoking & $18(23.7 \%)$ & $20(26.3 \%)$ & $38(25 \%)$ & 0.708 \\
\hline Pack-yrs (median) & 22.5 & 25 & 25 & 0.415 \\
\hline Cardial disease & $12(15.8 \%)$ & $14(18.4 \%)$ & $26(17.1 \%)$ & 0.667 \\
\hline Endocrine diseases & $23(30.3 \%)$ & $31(40.8 \%)$ & $54(35.5 \%)$ & 0.175 \\
\hline Diabetes & $11(14.5 \%)$ & $13(17.1 \%)$ & $24(15.8 \%)$ & 0.656 \\
\hline ASA score (1/2/3/missing) & $3 / 51 / 21 / 1$ & $0 / 45 / 28 / 3$ & $3 / 96 / 49 / 4$ & 0.114 \\
\hline Previous abdominal surgery & $17(22.4 \%)$ & $15(19.7 \%)$ & $32(21.1 \%)$ & 0.691 \\
\hline Neoadjuvant chemotherapy & $3(3.9 \%)$ & $3(3.9 \%)$ & $6(3.9 \%)$ & 1 \\
\hline Previous abdominal radiation & $2(2.6 \%)$ & $1(1.3 \%)$ & $3(2 \%)$ & 0.560 \\
\hline
\end{tabular}

ASA indicates American Society of Anesthesiologists; FEV1, forced expiratory 1-second volume; IPMN, intraductal papillary mucinous neoplasm; mITT, modified intention-totreat; $\mathrm{VC}$, vital capacity.

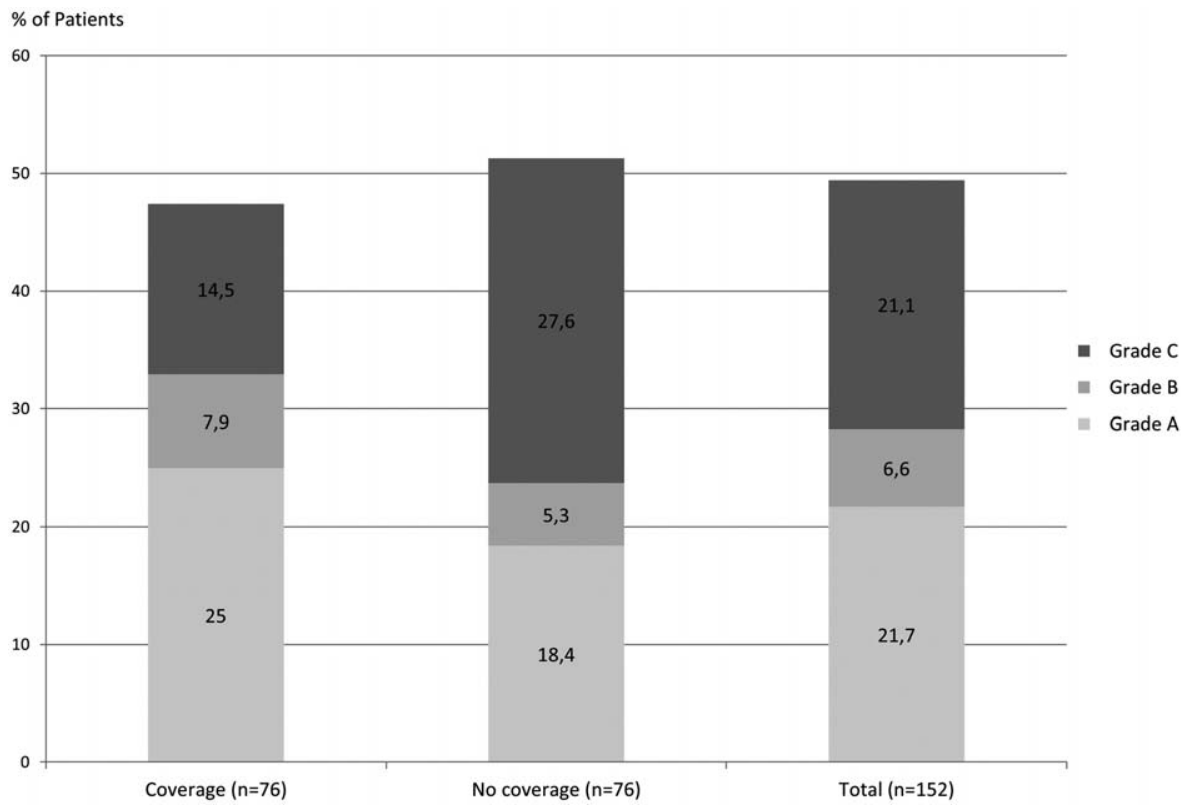

FIGURE 2. Postoperative pancreatic fistula (POPF) incidence and grading in the study groups. 
TABLE 2. Operative Parameters and Secondary Outcome (mITT)

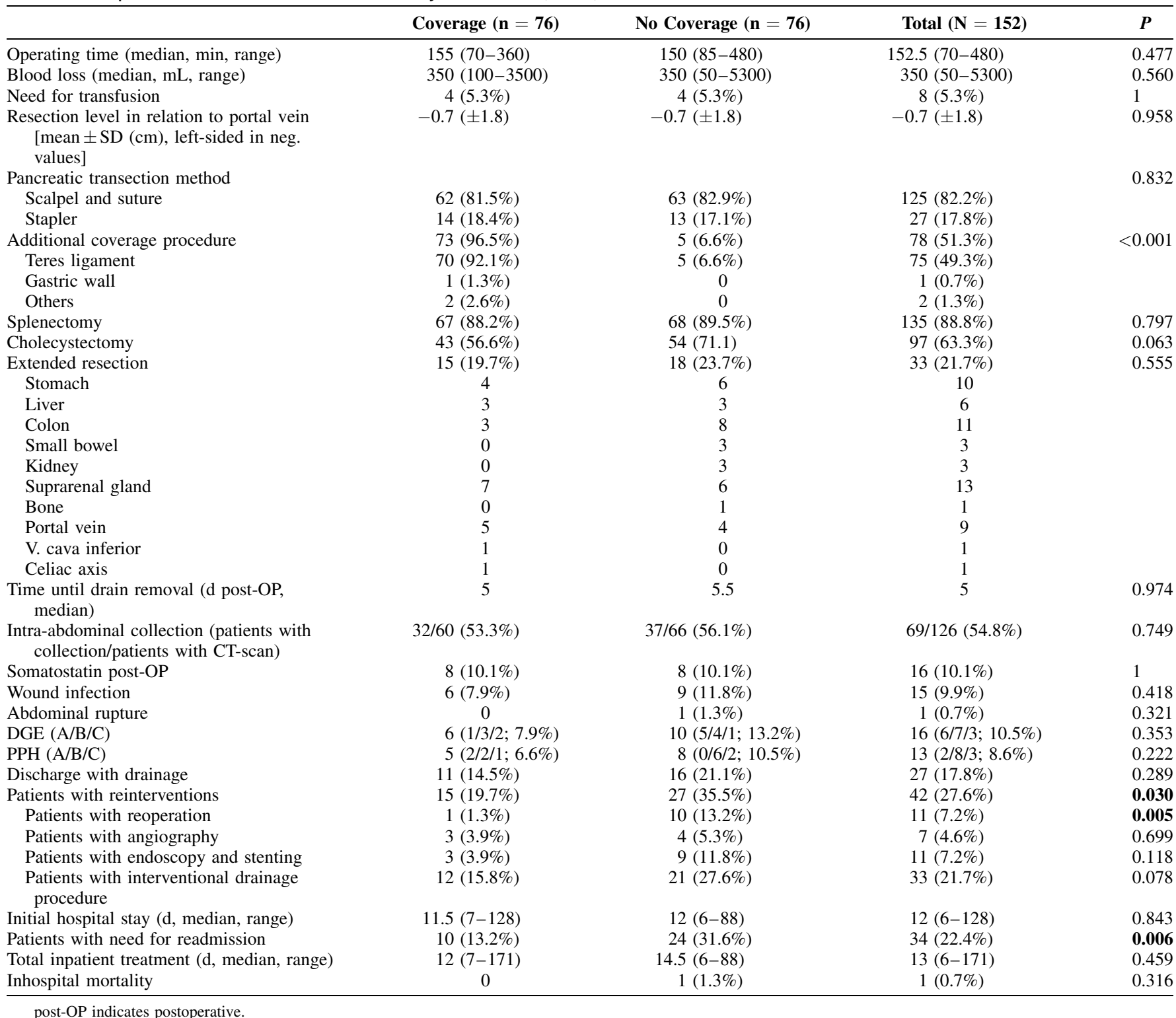

As POPF-related complications may occur delayed, ${ }^{6}$ the follow-up covered 40 days and 1 year after the index operation. Interestingly, this late follow-up revealed an additional 20 readmissions after the first follow-up. This observation has merely been described in other studies ${ }^{19}$ and raises the question if POPF rates may have been underestimated in past series with a limited follow-up time. Recent series are reporting significantly higher POPF rates of more than $50 \% .^{20,21}$

Apart from the medical impact of POPF and POPF-related complications, the associated higher healthcare costs of POPF patients without coverage are an important parameter in the outcome of DP. Although the cost calculation of the present study is not directly transferable to other hospitals due to the underlying nation and region-specific amounts, it can be assumed that the basic effect of reduced expenses for POPF therapy in the coverage group is transferable to other institutions and countries.
The teres ligament patch technique is safe and does not cause additional expenses or a relevant prolongation of the operation time. It has also been described to be useful for POPF prevention in pancreatic enucleations. ${ }^{22}$ In cases in which the teres ligament patch is not available for technical reasons (eg, tension due to an increased distance between abdominal wall and resection margin in obese patients or due to previous operations), use of gastric or jejunal wall may be an alternative to cover the pancreatic remnant. ${ }^{11,23}$ The supposed effect of the coverage procedure is an encapsulation of postoperative fluid secretion from the resection margin and prevention of a leakage towards the abdominal cavity. Interestingly, the drainage fluid did not show differences in secretion volume or enzyme concentration until postoperative day 4 between both groups. This may be explained by the process of wound healing, which takes several days for sealing the remnant by local inflammation and adhesion. Especially during the initial phase, fluid secretion can 
TABLE 3. Management of POPF Patients (ITT Population)

\begin{tabular}{|c|c|c|c|c|c|c|c|c|}
\hline & \multicolumn{2}{|c|}{ Initial Hospital Stay } & \multicolumn{2}{|c|}{ Readmissions } & \multicolumn{4}{|c|}{ Total Inpatient Treatment } \\
\hline & $\begin{array}{l}\text { Coverage } \\
(\mathbf{n}=\mathbf{3 6})\end{array}$ & $\begin{array}{l}\text { No coverage } \\
\quad(\mathbf{n}=\mathbf{3 9})\end{array}$ & $\begin{array}{l}\text { Coverage } \\
(\mathbf{n}=\mathbf{9})\end{array}$ & $\begin{array}{l}\text { No coverage } \\
\quad(\mathbf{n}=19)\end{array}$ & $\begin{array}{l}\text { Coverage } \\
(\mathbf{n}=\mathbf{3 6})\end{array}$ & $\begin{array}{l}\text { No coverage } \\
\quad(\mathbf{n}=\mathbf{3 9})\end{array}$ & $\begin{array}{c}\text { Total } \\
(\mathbf{N}=\mathbf{7 5})\end{array}$ & $\boldsymbol{P}$ \\
\hline Patients with reinterventions & $9(25 \%)$ & $13(33.3 \%)$ & $3(33.3 \%)$ & $11(57.9 \%)$ & $12(33.3 \%)$ & $24(61.5 \%)$ & $36(48 \%)$ & 0.014 \\
\hline Patients with reoperation & $1(2.8 \%)$ & $6(15.4 \%)$ & 0 & $3(15.8 \%)$ & $1(2.8 \%)$ & $9(23.1 \%)$ & $10(13.3 \%)$ & 0.009 \\
\hline Patients with angiography & $1(2.8 \%)$ & $2(5.3 \%)$ & $1(11.1 \%)$ & $1(5.3 \%)$ & $2(5.6 \%)$ & $3(7.7 \%)$ & $5(6.7 \%)$ & 0.715 \\
\hline $\begin{array}{l}\text { Patients with endoscopy and } \\
\text { stenting }\end{array}$ & $2(5.6 \%)$ & $6(15.4 \%)$ & 0 & $3(15.8 \%)$ & $2(5.6 \%)$ & $9(23.1 \%)$ & $11(14.7 \%)$ & 0.032 \\
\hline $\begin{array}{l}\text { Patients with interventional } \\
\text { drainage procedure }\end{array}$ & $7(19.4 \%)$ & $10(25.7 \%)$ & $3(33.3 \%)$ & $9(47.4 \%)$ & $10(27.8 \%)$ & $19(48.7 \%)$ & $29(38.7 \%)$ & 0.064 \\
\hline Patients with need for readmission & $\mathrm{X}$ & $\mathrm{X}$ & $\mathrm{X}$ & $\mathrm{X}$ & $9(25 \%)$ & $19(48.7 \%)$ & $28(27.3 \%)$ & 0.034 \\
\hline At least 2 readmissions & $\mathrm{X}$ & $\mathrm{X}$ & $\mathrm{X}$ & $\mathrm{X}$ & $1(2.8 \%)$ & $9(23.1 \%)$ & $10(13.3 \%)$ & 0.009 \\
\hline Hospital stay (d, median, range) & $12(7-90)$ & $15(6-88)$ & $5(1-7)$ & $7(1-21)$ & $15(7-90)$ & $25(6-88)$ & $21(6-90)$ & 0.162 \\
\hline Treatment costs (Euro, median) & $\mathrm{X}$ & $\mathrm{X}$ & $\mathrm{X}$ & $\mathrm{X}$ & 10138 & 13998 & 12737 & 0.035 \\
\hline
\end{tabular}

occur, which is finally stopped by the sealing effect. This pathophysiological mechanism may provide an additional explanation why the overall incidence of POPF- which is defined as a leakage on or after postoperative day 3-may not be altered by the procedure.

In the follow-up imaging, postoperative fluid collections at the resection site were observed in more than $50 \%$ of the patients. We have recently reported this high incidence of fluid collections after $\mathrm{DP}^{24}$ The vast majority of these collections are not clinically relevant. However, in patients who required drainage, amylase concentration of the fluid contained in these collections differed between covered and uncovered patients with remarkably lower amylase values after the coverage procedure. This gives another explanation for the observed divergent postoperative courses of fistula patients in both groups.

A recent randomized controlled trial in which a thin flap of the falciforme ligament was detached and sutured to the resection margin did not find any effect on POPF rate or on the postoperative course. ${ }^{25}$ Supposedly, a devascularized and rather small flap might be more prone to atrophy and necrosis during the postoperative period when sealing process takes place. A pedicled and vascularized flap, which was used in our DISCOVER trial, may be more effective.

In the present study, all procedures were carried out as open DPs according to the study protocol, which was drafted during a period of time when the laparoscopic approach was not yet regarded as the standard for DP. A laparoscopic use of the teres ligament patch technique is certainly possible as well. To date, there are no studies available for this approach. Based on the results of the DISCOVER trial, this technique should also be evaluated in laparoscopic DP as the problem of POPF is an unsolved issue in this setting as well. ${ }^{26}$

\section{CONCLUSIONS}

Coverage of the pancreatic remnant during DP is associated with less postoperative reinterventions, reoperations, and less need for readmission. Although overall fistula rate is not reduced by the coverage procedure, it should be considered as a valid possibility to prevent complications after DP as it is easy to perform and shows a clinical benefit.

\section{REFERENCES}

1. Reames BN, Ghaferi AA, Birkmeyer JD, et al. Hospital volume and operative mortality in the modern era. Ann Surg. 2014;260:244-251.

2. Hackert T, Buchler MW. Remnant closure after distal pancreatectomy: current state and future perspectives. Surgeon. 2012;10:95-101.

3. McMillan MT, Christein JD, Callery MP, et al. Comparing the burden of pancreatic fistulas after pancreatoduodenectomy and distal pancreatectomy. Surgery. 2015. In press.
4. Knaebel HP, Diener MK, Wente MN, et al. Systematic review and metaanalysis of technique for closure of the pancreatic remnant after distal pancreatectomy. Br J Surg. 2005;92:539-546.

5. Olah A, Issekutz A, Belagyi T, et al. Randomized clinical trial of techniques for closure of the pancreatic remnant following distal pancreatectomy. $\mathrm{Br} \mathrm{J}$ Surg. 2009;96:602-607.

6. Diener MK, Seiler CM, Rossion I, et al. Efficacy of stapler versus hand-sewn closure after distal pancreatectomy (DISPACT): a randomised, controlled multicentre trial. Lancet. 2011;377:1514-1522.

7. Probst P, Huttner FJ, Klaiber U, et al. Stapler versus scalpel resection followed by hand-sewn closure of the pancreatic remnant for distal pancreatectomy. Cochrane Database Syst Rev. 2015.

8. Iannitti DA, Coburn NG, Somberg J, et al. Use of the round ligament of the liver to decrease pancreatic fistulas: a novel technique. J Am Coll Surg. 2006;203:857-864.

9. Kleeff J, Diener MK, Z'Graggen K, et al. Distal pancreatectomy: risk factors for surgical failure in 302 consecutive cases. Ann Surg. 2007;245: $573-582$.

10. Ferrone CR, Warshaw AL, Rattner DW, et al. Pancreatic fistula rates after 462 distal pancreatectomies: staplers do not decrease fistula rates. J Gastrointest Surg. 2008;1691-1697 [discussion 1697-8].

11. Kuroki T, Tajima Y, Tsuneoka N, et al. Gastric wall-covering method prevents pancreatic fistula after distal pancreatectomy. Hepatogastroenterology. 2009;877-880.

12. Hassenpflug M, Hartwig W, Strobel O, et al. Decrease in clinically relevant pancreatic fistula by coverage of the pancreatic remnant after distal pancreatectomy. Surgery. 2012;152:164-171.

13. Bassi C, Dervenis C, Butturini G, et al. Postoperative pancreatic fistula: an international study group (ISGPF) definition. Surgery. 2005;138:8-13.

14. Hackert T, Hinz U, Pausch T, et al. Postoperative pancreatic fistula: we need to redefine grades B and C. Surgery. 2016;159:872-877.

15. Wente MN, Bassi C, Dervenis C, et al. Delayed gastric emptying (DGE) after pancreatic surgery: a suggested definition by the International Study Group of Pancreatic Surgery (ISGPS). Surgery. 2007;142:761-768.

16. Wente MN, Veit JA, Bassi C, et al. Postpancreatectomy hemorrhage (PPH): an International Study Group of Pancreatic Surgery (ISGPS) definition. Surgery. 2007; 142:20-25.

17. Hassenpflug M, Bruckner T, Knebel P, et al. DISCOVER trial: distal resection of the pancreas with or without coverage of the pancreatic remnant: study protocol of a randomised controlled trial. Trials. 2013;14:430.

18. Rodriguez JR, Germes SS, Pandharipande PV, et al. Implications and cost of pancreatic leak following distal pancreatic resection. Arch Surg. 2006;141:361-365 [discussion 366].

19. Ceppa EP, McCurdy RM, Becerra DC, et al. Does pancreatic stump closure method influence distal pancreatectomy outcomes? J Gastrointest Surg. 2015;19:1449-1456.

20. Montorsi M, Zerbi A, Bassi C, et al. Efficacy of an absorbable fibrin sealant patch (TachoSil) after distal pancreatectomy: a multicenter, randomized, controlled trial. Ann Surg. 2012;256:853-859.

21. Sa Cunha A, Carrere N, Meunier B, et al. Stump closure reinforcement with absorbable fibrin collagen sealant sponge (TachoSil) does not prevent pancreatic fistula after distal pancreatectomy: the FIABLE multicenter controlled randomized study. Am J Surg. 2015;210:739-748. 
22. Hackert T, Lozanovski VJ, Werner J, et al. Teres hepatitis ligament flap plasty to prevent pancreatic fistula after tumor enucleation. J Am Coll Surg. 2013;217:e29-e34.

23. Issekutz A, Belagyi T, Romics L, et al. [A novel technique for the closure of the pancreatic remnant using jejunal serosa following distal pancreatectomy] Magy Seb. 2006;59:117-121.

24. Tjaden C, Hinz U, Hassenpflug M, et al. Fluid collection after dista pancreatectomy: a frequent finding. HPB. 2016;18:35-40.

25. Carter TI, Fong ZV, Hyslop T, et al. A dual-institution randomized controlled trial of remnant closure after distal pancreatectomy: does the addition of a falciform patch and fibrin glue improve outcomes? J Gastrointest Surg. 2013;17:10210-10219.

26. Jusoh AC, Ammori BJ. Laparoscopic versus open distal pancreatectomy: a systematic review of comparative studies. Surg Endosc. 2012;26:904-913.

\section{DISCUSSANTS}

\section{O. Farges (Clichy, France):}

I congratulate you and your group for the persistent effort in finding technical ways to reduce the incidence of pancreatic fistula and for the quality of your presentation. I have 3 questions: First, this is an intent-to-treat analysis, but I notice that 8 patients ( 3 in the study group and 5 in the control group) did not receive the treatment they should have received. Do the results of the study change if patients are allocated to the treatment actually received or are excluded from analysis? Second, how many surgeons were involved and how did you ensure that the coverage procedure had been done properly? As this is a concern common to trials focusing on a technique, don't you think that, in the future, a picture should be taken at the end of surgery? Third, the main difference in the 2 groups is a reduced incidence of reoperation and of readmission in the study group. Both endpoints are somewhat subjective. As this is an open (not a blinded) study and since the previous study from your group showed that coverage of the pancreatic remnant decreased clinically relevant fistula, is there a risk that management was more conservative in the study group than in the control group?

\section{Response From T. Hackert (Heidelberg, Germany):}

We had this problem as 5 and 3 cross-over patients were not treated according to the protocol in each group. I think this problem can occur in every clinical study: that the patient is not compliant or the surgeon is not compliant. We did the intention-to-treat analysis because this was stated in the study protocol which I presented to you. After this point came up, during the revision of the manuscript, we also did an internal per-protocol analysis. This analysis basically showed the same results, which shows that the study was not very much influenced by these 3 and 5 patients.

I think the second question is an important topic in all surgical studies. We basically had 8 surgeons who were responsible during the study period between 2010 and 2014. We intended to take a photograph of every operation which was also stated in the study protocol and should have been done. Finally, we ended up with approximately $80 \%$ of photographs. In $20 \%$ of the patients, photographs were not present, but surgeons had to fill in an evaluation form after the operation and state that "yes, I did coverage" or "no, I did no coverage." In addition, the resection margin in relation to the portal vein was also documented.

I do not think fistula management was a bias, because the indication for an intervention or the indication for a reoperation should not be based on any study you just perform currently or that you think it will not become that severe. I think the clinical impression of the patient is what leads to the decision of "yes, he needs a drainage" or "no, he needs no drainage," and not any other confounding consideration.

\section{N. Senninger (Münster, Germany):}

You are creating a new gap by bending the teres ligament to the pancreas. Has this gap ever been producing interceptional power? I thought Heidelberg was a nondraining clinic. Did you change your strategy?

The omentum is next to it, and has much more important cells than this little fat pad of the teres ligament. Why did not you choose the teres ligament? By the way, many have tried that before and there was no benefit.

\section{Response From T. Hackert (Heidelberg, Germany):}

Regarding the "gap" that we create, of course there is the theoretical possibility that there might be some herniation. This is something we have never observed because it is located only in the upper abdominal space below the liver on the right side. I think the small bowel will not really come there by going behind the stomach. We had no revisions because of a problem like that.

We routinely place passive drainage tubes intraoperative after distal pancreatectomy is routinely drained as it was performed in the study. To use the omentum is of course a good possibility; however, I think the omentum does not have this tight and firm surface which the teres ligament has. The coverage material from the teres ligamentfrom my experience-is more suitable than the omentum, which is often a bit crispy and not solid. This does not nearly fit that well to cover the pancreatic cut surface.

\section{Bruns (Cologne, Germany):}

Was the texture of the pancreas only intraoperatively evaluated? For pancreatic stump closure, which technique did you allow: stapler or conventional surgical cutting and suturing, or any of them? Was the decision about the closure technique made intraoperatively?

\section{Response From T. Hackert (Heidelberg, Germany):}

The texture of the pancreas was evaluated intraoperatively; however, we did not document the texture in the study protocol. This may be a little shortcoming of the study.

Regarding the choice for stapler application, this was free to every surgeon. By that time we had used this adaptable stapler, which may also be suitable for a thicker pancreas, but actually for thick pancreas probably most surgeons choose to cut it by scalpel and close the cut margin by suture. Furthermore, crushing of the pancreas or squeezing the pancreas might cause a fistula and a hematoma when the stapler is not suitable due to the thickness of the parenchyma.

\section{A. Sauvanet (Clichy, France):}

One of the well-known risk factors of pancreatic fistula after distal pancreatic is body mass index (BMI). I did not notice that the BMI was strictly equivalent between both groups. My second question is about the way you plan the postoperative course after discharge. What was the proportion of patients who went back home, who went to other hospitals or other facilities? Was the course after hospital stay equivalent between the groups? It could have influenced the rate of readmission and the rate of reintervention, particularly interventional radiology.

\section{Response From T. Hackert (Heidelberg, Germany):}

The BMI was analyzed and it was not a confounding factor in the analysis, which is shown in the manuscript. The discharge policy of course plays a role for the readmission. Basically we tried to discharge the patients when they had no drain anymore in either group, no matter if covered or not covered. Of course, sometimes 
patients are also discharged with a drainage and this might certainly cause another readmission, as well as readmission without drainage was included when necessary for an additional new drainage. This policy, I think, was similar in both groups.

\section{A. Olah (Györ, Hungary):}

The reoperation rate was slightly high in the control group, more than $13 \%$. What was the major indication for the reoperation? Recently, we tried to increase the rate of laparoscopic distal pancreatectomy. Do you have any experience with this technique laparoscopically or only in open surgery?

\section{Response From T. Hackert (Heidelberg, Germany):}

Regarding the rate of reoperation, we had $13 \%$ in the controlgroup without coverage. Except for 1 patient, they were all fistula-associated, which means due to bleeding, due to infection control, due to any problems associated with a pancreatic fistula such as bowel perforation. I think this was quite impressive as in the teres group we only had 1 reoperation for fistulaassociated reasons.

Regarding the laparoscopic approach, we have, of course, in the meantime standardized distal pancreatectomy as a laparoscopic procedure. We have not yet performed the teres ligament patch laparoscopically, although this might be possible as well.

\section{T. van Gulik (Amsterdam, The Netherlands):}

You showed no significant differences in the rate of interventions required in the teres ligament patch group and no-coverage group. Regarding the protective effects of the teres ligament patch, do you think that it really prevents leakage or does it contain leakage? In other words, does coverage conceal leakage to result in less problems that need an intervention? The only way you could tell if that is the mechanism is by checking on follow-up imaging in these patients.

\section{Response From T. Hackert (Heidelberg, Germany):}

That's a very good question because we see a lot of fluid collections without a clinical impact after distal pancreatectomy of any kind. I think that from the mechanism, the teres ligament might cover, attach, and maybe have a fluid collection below it. This is usually not clinically relevant and does not cause any problems. As I showed you with regard to the peak amylase values, there is a certain leakage, of course, but it is not that severe as when the pancreatic remnant is not covered. 